\title{
Avoiding the misuse of fresh frozen plasma
}

\author{
No scientific basis for most of its uses; hospital transfusion committees should draw up guidelines
}

Studies on the use of fresh frozen plasma in Britain, where its administration has increased more than 10-fold in the past 15 years, and elsewhere have shown that it is often misused. ${ }^{1-3}$ Probably the main reasons for this are limited knowledge of its efficacy in specific situations, ignorance of its risks, and its increased availability. Guidelines on its use have recently been published by the British Committee for Standards in Haematology ${ }^{4}$; they complement those included in $A B C$ of Transfusion ${ }^{5}$ and the National Blood Transfusion Service's Handbook of Transfusion Medicine. ${ }^{6}$

Although fresh frozen plasma is widely advocated for patients with multiple coagulation defects associated with severe liver disease, disseminated intravascular coagulation, and massive blood transfusions, its effect is poorly defined. As a result, various ill supported practices persist, such as the transfusion of 2 units of fresh frozen plasma before liver biopsy when the prothrombin time is prolonged by 2-3 seconds. Large prospective controlled multicentre studies are needed to define the role of fresh frozen plasma in patients with acquired multiple coagulation defects, but the difficulties of undertaking such studies are formidable. What is clear is that if any benefit is to be obtained adequate quantities of fresh frozen plasma must be given of perhaps 4-6. donor units (about $800-1200 \mathrm{ml}$ ) over one or two hours-and then repeated. Fresh frozen plasma has no role as a volume expander; synthetic colloids are more effective, safer and cheaper. Fresh frozen plasma has no place in "formula" replacement protocols-for example, the administration of 1 unit of fresh frozen plasma after every 6 units of blood. Fresh frozen plasma should not be used to provide nutrition in patients with protein losing states. Its use as a source of immunoglobulins in the treatment of congenital immunodeficiency states has been superseded by use of intravenous immunoglobulin preparations.

Several reasons exist for avoiding the inappropriate use of fresh frozen plasma. Risks associated with its transfusion, particularly those of infection, should not be underestimated. The current estimated risk of acquiring HIV infection by transfusion in Britain is one in 3000000 donations; for hepatitis $B$ it is one in 20000 and for hepatitis $C$ it is less than one in 13000 (M Contreras and J Barbara, North London Blood Transfusion Centre, personal communication). Other adverse effects include allergic reactions; intravascular haemolysis of recipient red cells after infusion of $\mathrm{ABO}$ incompatible plasma; fluid overload; the formation of antibodies to donor granulocytes, leading to leucocyte aggregation in pulmonary vessels and acute lung injury, a syndrome known to be related to transfusion; and possibly immune suppression. Two other factors merit consideration. Firstly, in the past few years in Britain, despite efforts to recruit new donors, rates of blood donation have remained static and in some places have fallen. ${ }^{3}$ Secondly, most regional transfusion centres charge between $£ 22$ and $£ 28$ for a unit of fresh frozen plasma, containing about $180 \mathrm{ml}$. Doctors should take careful account of these factors before treating patients with fresh frozen plasma; inappropriate use of such a scarce resource is wasteful.

Firm indications for the use of fresh frozen plasma are few. It is useful in the replacement of, usually congenital, single procoagulant factor deficiencies (for example, factor $\mathrm{V}$ ) when a specific or combined factor concentrate is unavailable. It has been used as replacement treatment in patients with congenital deficiencies of the naturally occurring anticoagulants antithrombin III, protein $C$, and protein $S$. Antithrombin III concentrates are now widely available, and commercially made protein $\mathrm{C}$ concentrates have recently become available for clinical trial. Fresh frozen plasma has been used in patients with congenital deficiency of $\mathrm{C} 1$ esterase inhibitor who develop severe angio-oedema, but a $\mathrm{C} 1$ esterase inhibitor concentrate is available. The role of specific factor concentrates in various acquired deficiency states (such as antithrombin III concentrate in disseminated intravascular coagulation) is being assessed; if found to be effective they could obviate the use of fresh frozen plasma. Fresh frozen plasma may be used to reverse oral anticoagulation associated with serious bleeding if prothrombin complex and factor VII concentrates are not available (about a litre is required for an adult). ${ }^{7} \mathrm{~A}$ similar regimen should be used to treat haemorrhagic disease of the newborn and bleeding associated with malabsorption of vitamin $\mathrm{K}$. Used aggressively in large volumes (at least 3 litres/day), usually with plasma exchange, fresh frozen plasma is the mainstay of treatment in patients with thrombotic thrombocytopenia purpura and related syndromes. It should be used to correct depletion of coagulation factors in bleeding associated with thrombolysis.

Several steps could be taken to optimise the use of fresh frozen plasma. Firstly, the guidelines of the British Committee for Standards in Haematology emphasise the importance of implementing educational programmes outlining the benefits and complications of treatment with blood components. Such programmes have been shown to reduce both the total amount of fresh frozen plasma transfused and 
the number of units transfused inappropriately. ${ }^{8}$ Education, however, does not necessarily lead to a consistent change in clinical practice. ${ }^{9}$ The use of fresh frozen plasma is controlled locally and determined largely by existing practice. Hospital transfusion committees should develop local guidelines based on a national protocol to promote the appropriate use of blood and blood components ${ }^{7}$; to achieve success representatives of all specialties using blood transfusion as well as haematologists must be involved.

A widespread practice in need of modification is the transfusion of 2 units of fresh frozen plasma to an adult in specific circumstances. Little, if any, scientific foundation exists for this practice and it is difficult to imagine what clinical benefit might be expected from a dose of fresh frozen plasma that is too small to increase the activity of any plasma factor yet sufficient to expose the recipient to its risks. Secondly, as with other blood components and in accordance with the Department of Health's circular on record keeping and stock control ${ }^{10}$ and regulations on product liability, ${ }^{11}$ the transfusion of fresh frozen plasma should be fully documented in patients' notes. This would become particularly important if a recipient were to acquire, say, HIV infection, when it would be necessary to identify the donor and provide written evidence to justify the use of the blood component implicated.

Thirdly, the introduction of further safety measures to the collection, screening, and treatment of plasma should be considered. The risk of infection with hepatitis $B$ virus could probably be reduced if screening for antibody to hepatitis $B$ core antigen was added to the current programme of testing of blood donations. Recently, pasteurisation of human plasma has been shown to allow recovery of more than $80 \%$ of the activities of clotting factors and protease inhibitors while ensuring that lipid enveloped and non-enveloped viruses are largely inactivated. ${ }^{12}$ Such an approach is attractive, although it would reduce the already scarce supply of plasma. Some transfusion centres have introduced ways of collecting a larger volume of fresh frozen plasma (200 or $250 \mathrm{ml}$ ) than the average $180 \mathrm{ml}$ from a single donation, thus lessening the number of donors who contribute to the volume that a single recipient receives. For this reason too, plasma collected by apheresis could be used when large volumes of plasma care required-such as in thrombotic thrombocytopenic purpura.

Finally, in this and related disorders, efforts should be directed to identifying the factor(s) in fresh frozen plasma that produce benefit as this could lead to the development of a plasma fractionation product.

St Mary's Hospital Medical School,

HANNAH COHEN Senior lecturer in haematology

London W2 1PG

1 National Institutes of Health. Fresh frozen plasma; indications and risks. $\{A M A$ 1985;253:551-3.

2 Synder AJ, Gottschall JL, Menitove JE. Why is fresh frozen plasma transfused? Transfusion 1986;26:107-12.

3 Thomson A, Contreras M, Knowles S. Blood component treatment: a retrospective audit in five major London hospitals. 7 Clin Pathol 1991;44:734-7.

4 British Committee for Standards in Haematology. Guidelines for the use of fresh frozen plasma. Transfusion Medicine 1992;2:57-63.

5 Contreras M, ed. $A B C$ of Transfusion. 2nd ed. London: BMJ Publications Group, 1992.

6 McClelland DBL, ed. Handbook of transfusion medicine. London: HMSO, 1989.

British Committee for Standards in Haematology. Guidelines on oral anticoagulant: second edition. fClin Pathol 1990;43:177-83.

8 Barnette RE, Fish DJ, Eisenstaedt RS. Modification of fresh-frozen plasma transfusion practices through educational intervention. Transfusion 1990;30:253-7.

9 Brien WF, Butler RJ, Inwood MJ. An audit of blood component therapy in a Canadian general teaching hospital. Can Med Assoc 7 1989;140:812-5.

10 Department of Health and Social Security. DHSS requirement for record keeping and stock control. London: HMSO, 1984. (HC (84) 7.)

11 Consumer Protection Act 1987. London: HMSO, 1987.

12 Burnouf-Radosevich M, Burnouf M, Huart J. A pasteurised therapeutic plasma. Infusionstherapie 1992;19:91-4.

\section{The gene for Huntington's disease}

\section{History repeats itself}

The gene causing Huntington's disease was localised to a specific chromosomal region soon after "reverse genetics" (now called positional cloning) was developed. In large families Huntington's disease was found to cosegregate with a variation in DNA sequence on the distal short arm of chromosome $4 .{ }^{1}$ Any element of luck involved in mapping the gene for Huntington's disease quickly evaded researchers subsequently. It has taken intensive effort by countless investigators for 10 years to identify the gene mutation responsible for the disease. ${ }^{2}$

Why did it take so long? Progressing from genetic linkage to isolation of the mutant gene in any disease is a formidable task, as even close DNA markers are usually more than a million base pairs away from the disease gene. These difficulties were enhanced in Huntington's disease by the position of the gene, which made it difficult to identify flanking markers, an important step in positional cloning. In addition, data from critical families with important recombination events (crossovers) were initially misleading. ${ }^{3}$

Recent work focused on identifying genes within a 500 kilobase stretch of DNA and analysing them for mutations, and eventually one was identified with a sequence of tandemly repeated trinucleotides (CAG) at one end. This repeat sequence varies in length in normal subjects (between 9 and 34 trinucleotides) but is longer, occasionally containing as many as 100 trinucleotides, in virtually all patients with Huntington's disease. ${ }^{2}$ Some patients may have a repeat length in the normal range, but the accuracy of diagnosis in these subjects has not yet been reassessed. ${ }^{4}$

The messenger RNA for the gene for Huntington's disease is 10.4 kilobases in length and is expressed in many tissues. Its predicted product has a molecular mass of 348 kilodaltons and bears no resemblance to any known protein. ${ }^{2}$ It has been termed huntingtin, which does not trip easily off the tongue. Whether the trinucleotide repeat sequence is translated and forms part of the protein is not yet known. The next phase of unravelling the molecular pathogenesis of Huntington's disease-determining function and malfunction of huntingtin -is under way. There are many pressing questions, and the first of these relates to correlating genotype with phenotype. Analysing repeat length for such studies, and for clinical purposes, is easier now with protocols ${ }^{5-7}$ that are technically easier than the original method. ${ }^{2}$

Preliminary data suggested that the length of the trinucleotide repeat and the age at disease onset were inversely correlated. $^{2}$ This has since been confirmed in a series of 440 patients. ${ }^{4}$ One feature of Huntington's disease, which has long been recognised, is that patients whose disease has a very early onset nearly always have affected fathers. This has a molecular correlate. Paternally transmitted cases have a larger number of CAG repeats in the gene than those with affected 